\title{
As medidas governamentais para interiorização dos médicos estão tendo impacto sobre os estudantes?
}

\author{
Are the governmental measures to promote \\ medical practice in rural areas having an impact \\ on medicine students?
}

\begin{abstract}
Ana Beatriz Cardoso Pereira', Lana Carla Fernandes de Albuquerque', Luana Bastos de Mont'Alverne Ferreira', Luciano Moura de Assunção', Cezar Augusto Muniz Caldas²

'Faculdade de Medicina, Universidade Federal do Pará, Belém (PA), Brasil. ${ }^{2}$ Universidade Federal do Pará, Belém, (PA), Brasil.
\end{abstract}

\section{RESUMO}

Objetivo: Identificar a repercussão das políticas públicas para interiorização dos médicos e caracterizar o interesse dos alunos de Medicina em residir no interior. Métodos: Foi realizado um estudo transversal, descritivo e analítico, entre agosto de 2013 e dezembro de 2016, incluindo 381 concluintes de Medicina da Universidade Federal do Pará. Resultados: Do total, 26,2\% mostraram-se interessados em residir no interior, sendo o maior valor no primeiro semestre de 2014, com 35\%; e o menor, no segundo semestre de 2015, com 13,63\%. Conclusão: Notou-se uma tendência a aumento do desejo à interiorização, com valores estatisticamente significativos ao comparar os valores em 2014, 2015 e 2016. Fatores contribuintes incluem principalmente medidas governamentais para melhoria da qualidade da assistência nas cidades do interior.

palavras-chave: Educação médica. Assistência médica. Políticas públicas. Profissional da saúde. Atenção à saúde.

Recebido: Out. 13, 2017

Aceito: Abr. 23, 2018

\section{COMO CITAR ESTE ARTIGO}

Pereira ABC, Albuquerque LCF,

Ferreira LBM, Assunção LM, Caldas CAM. As medidas governamentais para interiorização dos médicos estão tendo impacto sobre os estudantes? Interdisciplinary Journal of Health ez:2(2):89-95. https://doi.org/10.4322/ijhe.2017.002

\section{CORRESPONDÊNCIA}

Cezar Augusto Muniz Caldas Instituto de Ciências da Saúde, Universidade Federal do Pará

Av. Generalíssimo Deodoro, 1, Bairro Umarizal, CEP 66050-160, Belém (PA) Brasil

Tel.: (91) 99915-7041

cezarcaldas@ufpa.br

\section{FONTE DE FINANCIAMENTO}

Houve financiamento pela

Pró-reitoria de Pesquisa e Pós-

graduação (PROPESP) através de

bolsa de iniciação científica.

\section{CONFLITO DE INTERESSE}

Os autores declararam não

haver conflitos de interesse.

O estudo foi realizado no Instituto de Ciências da Saúde, Universidade Federal do Pará, Belém (PA), Brasil.

\section{ABSTRACT}

Objective: Identify the repercussion of public policies to incentive medical practice in rural areas and characterize the interest of Medicine students to reside in rural areas. Methods: A transversal, descriptive and analytic study was carried out between August 2013 and December 2016, including 381 students that were completing Medicine degree in Federal University of Pará, Brazil. Results: From this total, 26.2\% showed interest in residing in rural areas, considering that the greatest value was in the first semester of 2014 (35\%) and the smallest in the second semester of 2015 (13.63\%). Conclusion: It was remarked a tendency to increase the aim to reside in rural areas, with statistically relevant values, when we compare the values in 2014, 2015 and 2016. The contributing factors include, mainly, governmental measures to improve the assistance quality in rural cities.

KEYWORDS: Medical education. Medical assistance. Public policies. Health professional. Health care. 


\section{Introdução}

Situações de carência e má distribuição geográfica de provedores de serviços, especialmente médicos, têm sido apontadas como problemas graves. Há grande concentração de profissionais de saúde e de escolas médicas nos grandes centros urbanos; tornando, portanto, difícil a provisão de serviços em áreas rurais e isoladas ou ainda em comunidades de pequeno e médio portes. Essa situação caracteriza um quadro de exclusão social que reforça as desigualdades e compromete a cidadania ${ }^{1}$.

A partir da percepção da gravidade deste quadro social, estratégias do governo foram instauradas a fim de reverter tal situação. Em 1993, foi proposto o Programa de Interiorização do Sistema Único de Saúde (PISUS), defendendo a interiorização de uma equipe mínima além do médico; seguida do Programa de Interiorização do Trabalho em Saúde (PITS) e os atuais Programa de Valorização do Profissional da Atenção Básica (PROVAB) e Programa Mais Médicos (PMM) ${ }^{2,3}$.

A discussão da temática tem sido levada à academia, visando formar profissionais que possam atuar nas comunidades mais distantes dos grandes centros urbanos. Sendo assim, procurando identificar a repercussão das políticas públicas para interiorização dos médicos, este estudo foi realizado visando caracterizar o interesse dos alunos do último ano do curso de Medicina em residir fora das capitais.

\section{Métodos}

Foi realizado um estudo transversal, descritivo e analítico, no período de agosto de 2013 a dezembro de 2016 para avaliar as impressões do aluno do último ano do curso de Medicina da Universidade Federal do Pará (UFPA) sobre a sua formação e caracterizar sua expectativa com relação à inserção no mercado de trabalho, pós-graduação e qualidade de vida. Dentre as expectativas pesquisadas, encontra-se o interesse por morar nas capitais ou em cidades do interior.

Foram incluídos estudantes do curso de Medicina da UFPA cursando o último ano, maiores de 18 anos e que aceitaram assinar o Termo de Consentimento Livre e Esclarecido (TCLE). Desta forma, foram incluídos, aleatoriamente, 381 alunos do último ano do Curso de Medicina da UFPA, sendo 64 do segundo semestre de 2013, 57 do primeiro semestre de 2014, 58 do segundo semestre de 2014, 49 do primeiro semestre de 2015, 44 do segundo semestre de 2015, 38 do primeiro semestre de 2016 e 71 do segundo semestre de 2016.

Após explicar sobre os objetivos, riscos e benefícios do estudo, os alunos que desejaram participar e aceitaram assinar o TCLE, responderam a um questionário elaborado pelos próprios autores, solicitando informações pessoais, questionando seu interesse por residir na capital ou no interior, dentre outros questionamentos.

Os dados recolhidos foram organizados e analisados através de planilhas do Microsoft Excel $2007^{\circledR}$. As variáveis categóricas foram apresentadas em valores absolutos e percentuais. As variáveis contínuas foram expressas como média e desvio padrão. A análise bivariada foi realizada pelo teste do Qui-quadrado e pelo teste Exato de Fisher. A análise de variáveis contínuas foi realizada pelo Teste T-Student. O processamento estatístico foi realizado no software BioEstat versão 5.4.

O estudo foi aprovado pelo Comitê de Ética em Pesquisa com número do parecer 319.222 (CAAE: 16134813.0.0000.0018). O curso de Medicina da UFPA é localizado em Belém-Pará, capital do estado, oferecendo 150 vagas anuais, com cerca de 75 concluintes por semestre.

\section{Resultados}

Entre o segundo semestre de 2013 e segundo semestre 2016 foram avaliados 381 acadêmicos. A maioria dos indivíduos era do gênero masculino, representando $58 \%$ do total; a média de idade foi de 26,21 anos; e o percentual de indivíduos 
interessados em residir em cidade do interior foi de $26,2 \%$. Não houve diferença estatística entre as médias de idade de acordo com o gênero e nem entre o desejo de residir no interior de acordo com o gênero (Tabela 1).

Uma minoria dos acadêmicos apresentou desejo de interiorização, com o maior valor de 35\%, no primeiro semestre de 2014, e menor valor de 13,63\%, no segundo semestre de 2015. Por outro lado, nota-se uma tendência a aumento do desejo por residir no interior, com valores estatisticamente significativos ao comparar os valores iniciais $\left(2^{\circ} / 2013: 15,63 \%\right)$ com $1^{\circ} / 2014(35 \%$; $p=0,0133), 1^{\circ} / 2015(32,65 \%$; $p=0,0331)$ e 2\% 2016 (32,39\%; $\mathrm{p}=0,0236)$ (Gráfico 1).

Tabela 1. Avaliação dos acadêmicos quanto gênero, idade e desejo à interiorização.

\begin{tabular}{|c|c|c|c|c|}
\hline Semestre/Ano & Gênero & $\mathbf{N}(\%)$ & $\begin{array}{c}\text { Idade }^{*} \\
\text { (Média } \pm \text { DP) }\end{array}$ & $\begin{array}{l}\text { Interior }^{\#} \\
\mathbf{N}(\%)\end{array}$ \\
\hline \multirow[t]{2}{*}{$2 \% / 2013$} & Feminino & $35(54,68)$ & $25,27 \pm 2,59$ & $5(14,28)$ \\
\hline & Masculino & $29(45,32)$ & $25,35 \pm 2,59$ & $5(17,24)$ \\
\hline \multirow[t]{2}{*}{$1^{\circ} / 2014$} & Feminino & $26(45,61)$ & $27,26 \pm 5,57$ & $10(38,46)$ \\
\hline & Masculino & $31(54,39)$ & $27,61 \pm 6,29$ & $10(32,25)$ \\
\hline \multirow[t]{2}{*}{$2^{\circ} / 2014$} & Feminino & $22(37,93)$ & $25,51 \pm 2,16$ & $4(18,18)$ \\
\hline & Masculino & $36(62,07)$ & $25,54 \pm 2,18$ & $10(27,77)$ \\
\hline \multirow[t]{2}{*}{$1^{\circ} / 2015$} & Feminino & $18(36,73)$ & $27,74 \pm 4,28$ & $6(33,33)$ \\
\hline & Masculino & $31(63,27)$ & $28,02 \pm 4,58$ & $10(32,25)$ \\
\hline \multirow[t]{2}{*}{$2^{\circ} / 2015$} & Feminino & $22(50)$ & $25,36 \pm 2,40$ & $3(13,63)$ \\
\hline & Masculino & $22(50)$ & $25,95 \pm 4,77$ & $3(13,63)$ \\
\hline \multirow[t]{2}{*}{$1^{\circ} / 2016$} & Feminino & $14(36,84)$ & $25,52 \pm 2,37$ & $4(28,57)$ \\
\hline & Masculino & $24(63,15)$ & $25,54 \pm 2,32$ & $7(29,16)$ \\
\hline \multirow[t]{2}{*}{$2^{\circ} / 2016$} & Feminino & $23(32,39)$ & $25,85 \pm 2,79$ & $8(34,78)$ \\
\hline & Masculino & $48(67,61)$ & $25,75 \pm 2,81$ & $15(31,25)$ \\
\hline TOTAL & & $381(100)$ & $26,21 \pm 3,96$ & $100(26,2 \%)$ \\
\hline
\end{tabular}

DP: Desvio-padrão; *Teste T-Student: $p>0,05$; "Teste Qui-quadrado ou Teste Exato de Fisher: $p>0,05$.

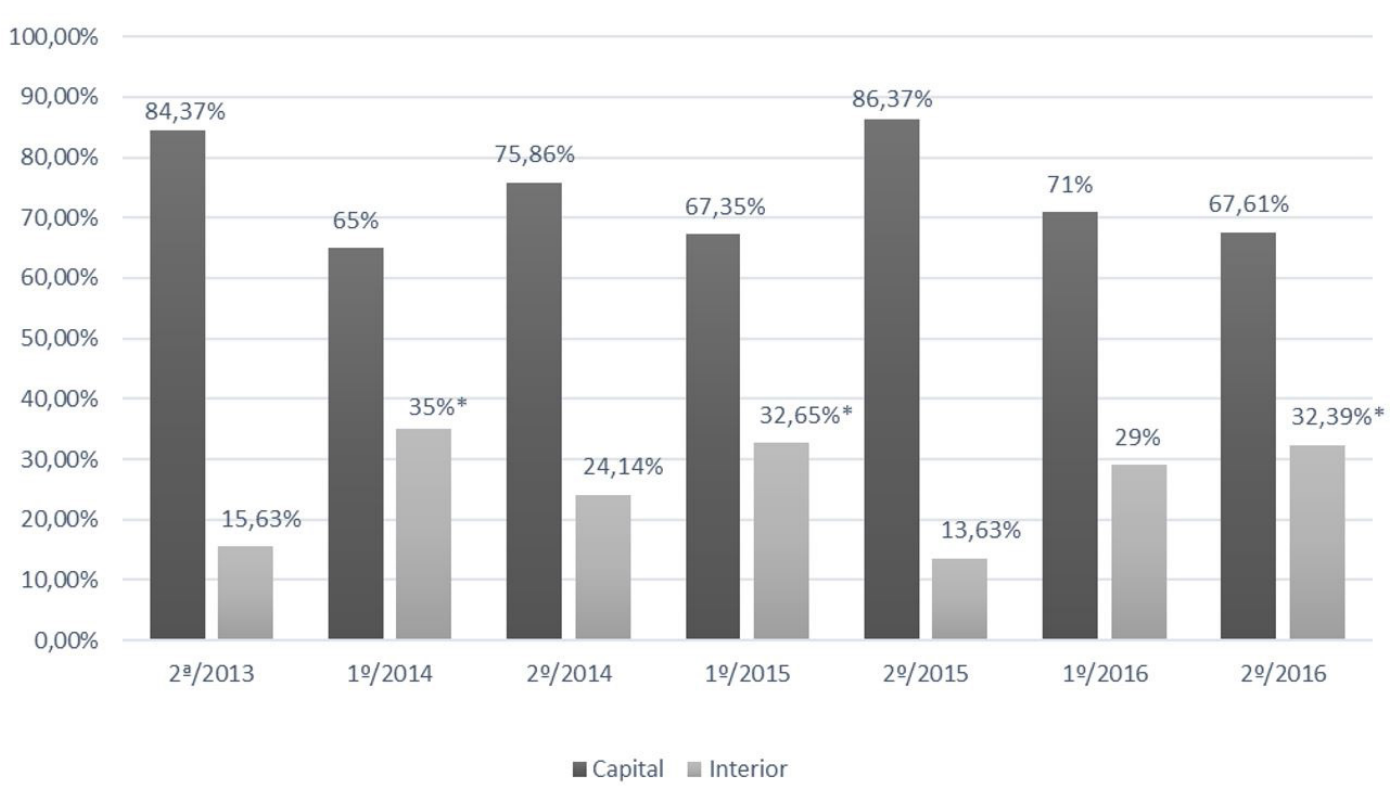

Gráfico 1. Avaliação dos acadêmicos quanto ao local onde deseja residir por semestre/ano. *Teste Qui-quadrado: $p<0,05$, em relação a 2\%/2013. 


\section{Discussão}

Embora não seja o desejo da maioria dos alunos estudados, observa-se um percentual crescente de estudantes com desejo de morar fora das capitais, podendo representar uma sinalização positiva às medidas governamentais que vêm sendo instituídas, assim como, podem representar resposta a fatores como violência e custo de vida, que podem acabar afastando médicos dos grandes centros urbanos.

As populações remotas rurais e pobres muitas vezes não são capazes de atrair ou reter profissionais de saúde. Há evidências de que um número crescente de profissionais pode ser mantido nas áreas rurais por meio de melhores políticas no setor de educação (escolas médicas e de enfermagem, políticas de admissão, bolsas de estudo e exigir um período de serviço em uma área rural após a formação completa) e no setor de saúde (pagamento de dificuldades, escolaridade para as crianças e planos de desenvolvimento de carreira profissional) $)^{4}$.

De fato, os médicos estão se concentrando nos grandes centros urbanos, deixando as cidades pequenas, áreas rurais e até as regiões mais pobres das grandes cidades desprovidas de assistência. Esse desequilíbrio na distribuição brasileira dos serviços de saúde ocorre em todos as esferas: entre áreas centrais e periféricas dentro das grandes cidades; entre áreas urbanas e rurais; entre capital e interior; e entre as macrorregiões ${ }^{5}$. A repartição é especialmente notável no âmbito da atenção primária, com falhas sistêmicas e, principalmente, profissionais com relutância em atender as comunidades rurais marginalizadas, ficando aquém das necessidades populacionais ${ }^{4}$.

O indicador mais utilizado, atualmente, para medir essas discrepâncias na distribuição do serviço médico é a relação de médicos por mil habitantes. O Brasil possui uma relação de 150 a 190 médicos por 100 mil habitantes, tendo uma relação média de um médico para cerca de 600 habitantes $^{6}$. Entretanto, essa média acoberta as desigualdades existentes no país, sendo os índices mais favoráveis nas regiões econômica e socialmente mais desenvolvidas. Corroborando a isso, aproximadamente $42 \%$ da população está assistida com menos de um médico para cada 4 mil habitantes. Além de que, $7 \%$ de todos os municípios brasileiros não registraram a presença de profissionais médicos nos limites de suas cidades 6 .

No Brasil, além da má distribuição existente entre capital e interior, persiste ainda a concentração da mão de obra médica nas regiões Sul e Sudeste. A região Sudeste, concentra $42,3 \%$ da população brasileira com $54,7 \%$ dos médicos, contrapondo-se à região Norte, com $8 \%$ da população, a qual detém apenas $4,3 \%$ desses profissionais ${ }^{6}$. Sendo esta, a região que possui os piores indicadores brasileiros, com uma menor disponibilidade de médicos e uma relação capital/interior igual à 6,3, ficando atrás apenas da região Nordeste, com esse índice igual à 10,3. Isso torna esta região uma área de maior vulnerabilidade, aumentando as dificuldades e o desejo de acesso médico ${ }^{5}$.

Em relação à região Norte, uma média de $26,9 \%$ dos médicos reside no interior, com valores mínimo e máximo de 6,9\% e 64,8\% no Amazonas e Tocantins, respectivamente. Nas capitais da Região Norte existem, em média, 2,5 médicos por mil habitantes, sendo esse valor de 3,4 para Belém-PA. Entretanto, no interior, essa relação é bem inferior, havendo apenas 0,3 médico por mil habitantes ${ }^{5}$.

Estes índices corroboram com os resultados obtidos neste presente estudo na medida em que, apesar de uma notória tendência ao aumento da vontade de interiorização, o percentil destes ainda é bem inferior ao dos que preferem continuar na capital. Pode-se inferir que o aumento do interesse, ainda que pequeno, do acadêmico do último ano em residir no interior tem relação direta com as mais diversas políticas públicas desenhadas pelo governo a fim de incentivar este "êxodo urbano" do médico recém-formado.

Sabe-se da criação destas iniciativas governamentais com o objetivo de atrair e fixar profissionais da saúde em regiões mais distantes das capitais ${ }^{7}$, como Programa de Interiorização das Ações de Saúde e Saneamento (PIASS), Programa de Interiorização 
do Sistema Único de Saúde (PISUS), Programa de Interiorização do Trabalho em Saúde (PITS), Programa de Valorização dos Profissionais da Atenção Básica (PROVAB) e o mais recente, Programa Mais Médicos (PMM) ${ }^{8-10}$.

Estes três últimos (PITS, PROVAB e PMM) começaram a apresentar mudanças na estratégia de atração profissional, visando incentivos financeiros e principalmente educacionais, com cursos de pós-graduação e entrada facilitada nos programas de residência médica, o que se mostra como uma medida particularmente atrativa para o grupo estudado, o formando, que vê nestes uma oportunidade para garantir renda salarial inicial e ainda iniciar sua especialização ${ }^{11}$.

Em 2001, o PITS propunha-se a estimular a ida de médicos e enfermeiros para os municípios mais carentes e distantes para que atuassem em equipes de Saúde da Família, por meio de incentivos financeiros e de formação profissional. Ficou ativo até 2004, sendo então encerrado no governo Lula ${ }^{11}$.

O PROVAB, a partir de 2012, consegue levar médicos, enfermeiros e dentistas para algumas das áreas mais necessitadas, no intuito de atuarem na atenção básica. A adesão ao primeiro ano de programa foi baixa, principalmente no que tange ao número de médicos contratados pelos municípios ${ }^{12}$. Em 2013, o programa enfocou o provimento de médicos, trazendo modificações na forma de contratação, fortalecimento da supervisão prática e estímulo ao curso de pós-graduação em atenção básica. Além de manter a bonificação para acesso aos Programas de Residência Médica. Deste modo, a adesão ao programa cresceu, mas ainda de forma insuficiente para as deficiências do país ${ }^{11}$.

Apesar da contribuição para atrair profissionais para áreas menos privilegiadas, os programas mencionados anteriormente não tiveram a magnitude do atual PMM. Este programa, introduzido no Brasil em julho de 2013, após os protestos populacionais nomeados de "Jornadas de Junho", constitui-se em uma política mais ampla que o PROVAB, inclusive incorporando-o ${ }^{7,11}$.

O PMM foi criado como uma medida de resposta imediata e emergencial para a formação e o provimento de médicos aos serviços de Atenção Básica. Este programa visa medidas estruturais de médio e longo prazos, que pretendem intervir na formação de médicos no Brasil, desde a abertura de novas vagas de graduação e residência médica, até a reorientação da formação de médicos e especialistas conforme as necessidades do SUS ${ }^{13}$.

Quanto ao eixo educacional, um forte componente de fixação dos médicos nos municípios, especialmente no interior, seria a disponibilidade de programas de graduação e residência médicas. A Política Nacional de Expansão das Escolas Médicas das Instituições Federais de Educação Superior, instituída no âmbito do PMM, tem como finalidades a criação de novos cursos de graduação em medicina e o aumento de vagas nos cursos atualmente existentes.

O plano de expansão do ensino médico está orientado por uma série de diretrizes, dentre as quais se destacam a diminuição das disparidades regionais, a interiorização e a adoção de estratégias de aperfeiçoamento da qualidade dos cursos. Com base nessas diretrizes, a partir de 2013 foram criadas 3.848 vagas em 60 cursos, sendo 3.179 em campi interiorizados e 669 vagas em cursos existentes nas capitais ${ }^{13}$. As vagas criadas até o momento já inverteram a concentração de escolas médicas em capitais e agora o interior do Brasil oferta mais vagas que as capitais, excetuando-se a região Norte do país, onde ainda há um predomínio de ofertas para a capital, como é o caso do curso de Medicina da UFPA ${ }^{13,14}$.

Quanto à oferta de programas e vagas de residência médica no Brasil, estas mostram-se deficitárias, havendo uma nítida concentração de vagas nas grandes cidades e regiões mais desenvolvidas. Para que o Governo Federal tivesse condições de dimensionar a necessidade de planejar a quantidade e a formação de médicos especialistas, a Lei que instituiu o Mais Médicos determinou a criação do Cadastro Nacional de Especialistas (CNE). Esse cadastro auxiliará o Governo Federal na tarefa 
de identificar a necessidade de médicos especialistas por região de saúde e de acordo com as necessidades da população ${ }^{13}$.

Dentro de uma perspectiva de ampliação do quadro médico nas unidades de atendimento à saúde foi estimado que, à época do lançamento do PMM, a proporção desses profissionais por habitantes era de 1,8 médico/1.000 habitantes, pretendendo-se assim atingir, em 2026, a marca de 2,7 médicos/1.000 habitantes ${ }^{13}$. Nota-se que a interiorização dessas iniciativas poderá proporcionar um aumento significativo nessa estimativa, ampliando o acesso à saúde básico e garantindo atuação dos médicos nas demais regiões do Brasil. Dessa forma, a implementação da residência médica de forma interiorizada propiciará benefícios, sobretudo, à população, tais como: aumento da proporção de médicos; redução de custos com deslocamentos para as capitais; maior acesso às especialidades médicas e; retenção de médicos na Unidade Básica de Saúde.

Há ainda em curso em algumas universidades do país, incluindo a UFPA, o chamado "Internato Rural", cujo objetivo é inserir gradativamente o acadêmico de Medicina em municípios menores. Devidamente tutelados e preceptorados, poderá resultar em relevante aprendizado e experiência profissional, além de proporcionar a integração com a comunidade, observação dos problemas locais, troca de informações com profissionais já atuantes no município, o que permitirá a cada acadêmico participante o reconhecimento do seu possível lugar nessa cadeia, identificando as potencialidades e os limites de sua atuação individual. A principal proposta do internato rural é, portanto, regimentar em forma de internato, acadêmicos de Medicina no Serviço de Saúde Pública Municipal de cidades onde atuam os profissionais do Programa Mais Médicos, sendo assim, mais uma forma de despertar o interesse à interiorização e melhor distribuição dos profissionais de saúde nas pequenas cidades ${ }^{15}$.

No presente estudo, apesar do predomínio do interesse em morar em capitais, nota-se uma redução de patamares acima de $80 \%$, para patamares em torno de $60 \%$. Paralelamente, o interesse em residir em cidades do interior, que iniciou como interesse de cerca de $15 \%$ dos entrevistados, ascendeu para valores acima de $30 \%$, remetendo a questionamentos e necessidade de estudos sobre quais fatores podem estar contribuindo para aumentar esse desejo por residir fora das capitais, ou mesmo, se haveriam fatores negativos, reduzindo o interesse dos futuros médicos por residir nas capitais.

A experiência de outros países demonstra que a competitividade cada vez maior do mercado de trabalho nos grandes centros urbanos, gradativamente, acarretaria em maior movimento migratório para cidades do interior que ofereçam condições para o exercício da Medicina e melhor qualidade de vida para os profissionais ${ }^{16-19}$. Além disso, estudos demonstraram que os aspectos atrativos que podem manter os profissionais atuando nessas regiões são: menor taxa de violência; menos poluição; desejo de mudança; sentimentos altruístas, como a atuação profissional junto às comunidades carentes, destacando ainda as condições de remuneração, incentivos financeiros, a disponibilidade de educação médica continuada; as oportunidades educacionais para os filhos e a proximidade da família e dos amigos ${ }^{20-22}$.

Apesar de esclarecer a respeito do crescente interesse nas vantagens oferecidas pelos programas de interiorização, este estudo deixa em aberto se, apesar do desejo em residir no interior, este realmente se concretizará após a formatura, ou seja, se as medidas implementadas pelo governo têm sido suficientes para fixar o médico em cidades do interior, oferecendo os aspectos citados anteriormente, como: qualidade de vida, boas condições de trabalho e moradia, e a tão almejada oportunidade para realização dos programas de residência médica.

\section{Conclusões}

De acordo com a análise da amostra desta pesquisa, pode-se inferir que uma mudança pode estar ocorrendo no interesse dos futuros médicos por residir fora dos grandes centros urbanos. Diversos fatores certamente contribuem para este movimento, desde 
medidas governamentais para melhoria da qualidade da assistência nas cidades do interior, contribuindo para o adequado exercício da Medicina, assim como, oportunidades e vivências oferecidas ainda durante o curso, que podem fazer o aluno, futuro médico, refletir sobre outras maneiras de desenvolver sua carreira profissional, chegando a comunidades que, até então, ficaram marginalizadas com relação ao acesso à saúde.

\section{Referências}

1. Brasil. Ministério da Saúde. Política de recursos humanos em saúde. Brasília: Ministério da Saúde; 2002.

2. Maciel R Fo, Branco MAF. Rumo ao interior: médicos, saúde da família e mercado de trabalho. Cad Saude Publica. 2009;21(11):2531-5.

3. Angotti H. No. Políticas de interiorização do médico brasileiro. Ibér Rev Interdisciplin Estud Ibér e Iber.-am. 2013;7(21):1-17.

4. Crisp MAN, Chen MDL. Global supply of health professionals. N Engl J Med. 2014;370(10):950-7. http://dx.doi.org/10.1056/ NEJMra1111610. PMid:24597868.

5. Silveira R, Pinheiro R. Entendendo a necessidade de médicos no interior da Amazônia - Brasil. Rev Bras Educ Med. 2014;38(4):451-9. http://dx.doi.org/10.1590/S0100-55022014000400006.

6. Campos FE, Machado MH, Girardi SN. A fixação de profissionais de saúde em regiões de necessidades. Divulg Saúde Debate. 2009;(44):13-24.

7. Oliveira FP, Vanni T, Pinto HA, et al. Mais Médicos: um programa brasileiro em uma perspectiva internacional. Interface (Botucatu). 2015;19(54):623-34. http://dx.doi.org/10.1590/1807-57622014.1142.

8. Mendes EV. As políticas de saúde no Brasil nos anos 80: A conformação da reforma sanitária e a construção da hegemonia do projeto neoliberal. In: Mendes, EV. Distrito Sanitário, o Processo Social de Mudança das Práticas Sanitárias do Sistema Único de Saúde. São Paulo: Hucitec; 1993. p. 19-91.

9. Rosas EJ.A extensão da cobertura dos serviços de saúde no Brasil: PIASS - análise de uma experiência [tese]. Rio de Janeiro (RJ): Escola Nacional de Saúde Pública/Fundação Oswaldo Cruz; 1981.

10. Maciel R Fo. Estratégias para a distribuição e fixação de médicos em sistemas e nacionais de saúde: o caso brasileiro [tese] Rio de Janeiro (RJ): Universidade do Estado do Rio de Janeiro; 2007.

11. Castro TFD. Reflexões sobre a prática de supervisão no programa de valorização do profissional da atenção básica (Provab) e no programa mais medicos [tese]. Campinas (SP): Faculdade de Ciências Médicas da Universidade Estadual de Campinas; 2015.

12. Carvalho MS. Programa de Valorização dos Profissionais da Atenção Básica: um olhar implicado sobre sua implantação [tese]. Brasilia (DF): Universidade de Brasília; 2013.

13. Brasil. Ministério da Sáude. Secretaria de Gestão do Trabalho e da Educação na Saúde (BR). Programa Mais Médicos - Dois anos: Mais Saúde para os Brasileiros. Brasília (DF): Ministério da Sáude; 2015.

14. Brasil. Ministério da Educação. Secretaria de Educação Superior. A democratização e expansão da educação superior no pais 2003-2014. Brasília: MEC/SESU; 2015.

15. Odorizzi VF, Marquezan RF, Odorizzi ALC; Noá KCA, Cruz AO. Internato Rural em Saúde Coletiva com ênfase em Saúde da Família e Programa Mais Médicos: Uma proposta de integração para a formação médica. Tempus Actas de Saúde colet. 2015; 9(4):115-22.

16. Newhouse J, Williams A, Bennett B, Schwartz W. Does the geographical distribution of physicians reflect market failure? Bell J Econ. 1982;13(2):493-506. http://dx.doi.org/10.2307/3003469.

17. Nocera S, Wanzenried G. On the dynamics of physician density; theory and empirical evidence for Switzerland. Diskussionsschriften. 2002:2-25.

18. Schwartz WB, Newhouse JP, Bernett BW, Williams AP. The changing geographic distribution of board-certified physicians: facts, theory and implications. Santa Monica, C. A.: The Rand Corporation; 1980.

19. Williams AP, Schwartz WB, Newhouse JP, Bennett BW. How many miles to the doctor? N Engl J Med. 1983;309(16):95863. http://dx.doi.org/10.1056/NEJM198310203091606. PMid:6621624.

20. Szafran O, Crutcher RA, Chaytors RG. Location of family medicine graduates' practices. What factors influence Albertans' choices? Can Fam Physician. 2001;47:2279-85. PMid:11768926.

21. Leonardson G, Lapierre R, Hollingsworth D. Factors predictive of physician location. J Med Educ. 1985;60(1):37-43. PMid:3965722.

22. Page J, Allison M, Andrade S, et al. Factors influencing medical interns trained at U.W.I. to work subsequently in a rural area in Jamaica. West Indian Med J. 1992;41(2):75-8. PMid:1523838.

\section{Contribuição dos autores}

Ana Beatriz Cardoso Pereira, Lana Carla Fernandes de Albuquerque, Luana Bastos de Mont'Alverne Ferreira e Luciano Moura de Assunção participaram de todas as etapas da pesquisa (revisão de literatura, elaboração de protocolos, coleta de dados e análise dos resultados) e da confecção do manuscrito. Cezar Augusto Muniz Caldas participou da concepção inicial, acompanhamento, orientação do estudo e elaboração do manuscrito. 